\title{
Quanto funziona il cortisone per os nel broncospasmo acuto in età prescolare? Risultati non confortanti di un RCT
}

Wallace A, Sinclair O, Shepherd M, et al.

Impact of oral corticosteroids on respiratory outcomes in acute preschool wheeze: a randomised clinical trial Arch Dis Child. 2021;106(4):339-344

La guida 2021 della Global Initiatve for Asthma (GINA) afferma che l'uso di steroidi per via orale nei bambini in età prescolare con broncospasmo acuto è raccomandato solo in caso di esacerbazione grave, tuttavia la letteratura continua a presentare risultati contrastanti. Lo Studio Wheeze and Steroids in Preschoolers (WASP), svolto in 3 pronto soccorso della Nuova Zelanda, ha randomizzato 477 bambini di età 24-59 mesi con broncospasmo acuto a ricevere prednisolone orale per 3 giorni vs placebo. Gli esiti respiratori misurati sono risultati contrastanti: il cambiamento del punteggio di gravità (PRAM) a 24 ore non è risultato differente tra i 2 gruppi, mentre il valore assoluto dello score sia a 4 che a 24 ore (esiti secondari) è risultato significativamente inferiore nel gruppo prednisolone. Anche il tasso di ricovero, la necessità di ulteriore trattamento con prednisolone orale e uso di farmaci endovena erano più bassi nel gruppo prednisolone. Si discute se il disegno di equivalenza dello studio e i margini scelti dagli autori per l'outcome primario abbiano attenuato la differenza tra i trattamenti.

How does oral cortisone work in acute bronchospasm in preschool age? ARCT with not reassuring results.

The 2021 guide of the Global Initiatve for Asthma (GINA) states that the use of oral steroids in preschool children with acute bronchospasm is only recommended in cases of severe exacerbation, however the literature continues to present conflicting results. The Wheeze and Steroids in Preschoolers (WASP) Study, conducted in 3 New Zealand emergency rooms, randomized 477 children aged 24-59 months with acute bronchospasm to receive oral prednisolone for 3 days versus placebo. The respiratory outcomes measured were conflicting: the change in severity score (PRAM) at 24 hours was not different between the 2 groups, while the absolute value of the score at both 4 and 24 hours (secondary outcomes) was significantly lower in the prednisolone group. Hospitalization rate, need for further oral prednisolone treatment, and intravenous drug use were also lower in the prednisolone group. It is debated whether the equivalence design of the study and the margins chosen by the authors for the primary outcome have attenuated the difference between the treatments.

\section{Metodo}

\section{Obiettivo (con tipo studio)}

Valutare se la somministrazione di prednisolone per via orale nei bambini in età prescolare con episodio di broncospasmo acuto modifica l'outcome respiratorio. Studio di equivalenza placebo-controllo, randomizzato, doppio cieco.

\section{Popolazione}

477 bambini di età compresa tra i 24 ed i 59 mesi con broncospasmo acuto associato a qualsiasi infezione respiratoria, arruolati in tre pronto soccorso della Nuova Zelanda.

Criteri di esclusione: pazienti con problematiche non respiratorie e concomitante broncospasmo; impossibilità ad avere un follow-up a 18-38 ore; terapia steroidea nei 7 giorni precedenti; PRAM (Preschool Respiratory Assessment Measure) score iniziale $<3$; malattia cardiaca o respiratoria cronica; storia di inalazione di corpo estraneo; storia di asma molto grave presente o pregressa; controindicazione a terapia steroidea; precedente arruolamento.

Tutti i pazienti, una volta eleggibili e definito il PRAM score, ricevevano un trattamento standard con $600 \mathrm{mcg}$ di salbutamolo inalatorio con distanziatore a distanza di $20 \mathrm{~min}$ per 3 somministrazioni. A seguire, dopo aver firmato il consenso di adesione allo studio, i pazienti venivano randomizzati a ricevere il trattamento o il placebo.

\section{Intervento}

238 pazienti hanno ricevuto prednisolone $(2 \mathrm{mg} / \mathrm{kg}$, massimo 40 $\mathrm{mg}$ ) per via orale una volta al giorno per 3 giorni consecutivi.

\section{Controllo}

239 pazienti hanno ricevuto placebo.

\section{Outcome/Esiti}

Obiettivo primario: cambiamento dello score PRAM rispetto al basale dal tempo di assunzione del trattamento a 24 ore di distanza (valutato con visita in presenza).

Obiettivi secondari: PRAM score a 4 ore; tasso di ricovero; durata dell'osservazione in pronto soccorso e dei ricoveri; dosaggio di salbutamolo somministrato a $48 \mathrm{~h}$ e 7 giorni; trattamento con dose aggiuntiva di prednisolone; tempo intercorso per la ripresa delle normali attività; eventi avversi compresa la necessità di trattamento medico endovenoso, di ricovero in terapia intensiva, di rivalutazione clinica per sintomatologia respiratoria in pronto soccorso o dal medico di famiglia nei 7 giorni successivi (valutati tramite interviste telefoniche).

Lo score PRAM si basa su 12 criteri clinici per la valutazione 
della severità e della risposta al trattamento del quadro di broncospasmo con un punteggio che va da un minimo di 0 (quadro lieve) a 12 - (quadro grave) (Tabella).

\section{Tempo}

Da Agosto 2014 a Settembre 2016, con un follow-up a 7 giorni.

\section{Risultati principali}

Non è stata riscontrata una differenza statisticamente significativa nel cambiamento dello score PRAM tra i due gruppi a 24 ore dalla somministrazione del farmaco (differenza media -0.39 , IC 95\% -0.84, 0.06, $\mathrm{p}=0.09$ ). Lo score PRAM assoluto è risultato essere più basso nel gruppo prednisolone a 4 ore $(p=0.01)$ e 24 ore $(\mathrm{p}=0.01)$, invece i sintomi hanno mostrato risoluzione per la maggior parte dei bambini indipendentemente dal trattamento intrapreso. Il tasso di ricovero, la necessità di terapia steroidea aggiuntiva per via orale, utilizzo di trattamenti endovenosi è risultato essere più basso nel gruppo prednisolone, nonostante non sia stata notata una differenza tra i due gruppi per quanto riguarda il tempo necessario per il ritorno alle normali attività o la necessità di una nuova valutazione clinica nei 7 giorni successivi. La dose di salbutamolo somministrata nelle prime $48 \mathrm{~h}$, la durata del ricovero, il tasso di riprensentazione in pronto soccorso non venivano influenzati dalla terapia steroidea. Mentre la durata della degenza in reparto e in pronto soccorso è risultata statisticamente significativa anche se borderline $(\mathrm{p}=0.06)$.

\section{Conclusioni}

Il trattamento con prednisolone per via orale non modifica l'evoluzione clinica a 24 ore e nelle ore successive nei bambini in età prescolare che presentano broncospasmo acuto.

\section{Altri studi sull'argomento}

Sebbene l'utilizzo degli steroidi per via orale sia ormai consolidato nel trattamento dell'asma acuto in età adulta e nei bambini più grandi, si hanno ancora dati contrastanti circa il suo utilizzo nel trattamento del broncospasmo in età prescolare. Nel 2019 una revisione sistematica e metanalisi ha incluso RCT riguardanti l'utilizzo di corticosteroidi rispetto al placebo nei bambini $<6$ aa con broncospasmo acuto. Quattro trial sono risultati eleggibili e gli autori hanno concluso che la terapia corticosteroidea per via orale (OCS) non riduceva il rischio di ospedalizzazione (Risk ratio 0.94 , IC 95\%CI 0.80, 1.12). Dei tre studi che riportano il tempo di permanenza in ospedale (dove viene identificata una differenza di 4 ore come clinicamente rilevante) due hanno riportato una differenza statistica ma non clinicamente rilevante (placebo 9 ore [IQR 2-16] vs OCS 6.2 [2-11.8]; e placebo 7.7 ore [5.0-22.9] versus OCS 6.8 [4-14]). Lo studio conclude quindi, con un'alta qualità di evidenza, che i corticosteroidi per via orale non dovrebbero essere usati in pronto soccorso in pazienti con broncospasmo in età prescolare. Gli effetti sui pazienti con asma nota o ospedalizzati con malattia severa è incerto [1]. In uno studio randomizzato placebo-controllo Foster et al hanno evidenziato una riduzione dei tempi di osservazione in pronto soccorso (di circa 3 ore) dei bambini di 24-72 mesi con broncospasmo acuto associato a forme infettive virali trattati con prednisolone rispetto al gruppo trattato con placebo. Da sottolineare come in questo studio l'effetto della terapia steroidea per os sulla durata dell'ospedalizzazione sia risultato maggiore nei pazienti giunti in pronto soccorso con un quadro di broncospasmo severo [2]. $\mathrm{Al}$ contrario, in un altro studio randomizzato placebo-controllo condotto su 687 bambini di 10-60 mesi con broncospasmo acuto associato a infezioni, l'utilizzo del prednisolone per via orale non si è dimostrato superiore al placebo nel ridurre i tempi di ospedalizzazione o nel ridurre il punteggio PRAM. Tuttavia in questo studio sono stati inclusi un numero considerevole di bambini molto piccoli con un probabile quadro di bronchiolite, su cui il cortisone non ha efficacia [3]. Un editoriale su JACI mette a confronto questi due studi dagli esiti contrastanti e propone alcune motivazioni alla base di queste differenze: 1) la severità degli episodi, nell'RCT di Foster et al. sono stati inclusi episodi più gravi che hanno più probabilità di migliorare in seguito alla somministrazione della terapia steroidea; 2) in Foster et al. sono stati esclusi i bambini $<2$ anni arricchendo quindi la popolazione in studio di bambini asmatici o predisposti ad avere asma in futuro e che quindi beneficiano in misura maggiore all'utilizzo di corticosteroidi [4]. Una metanalisi condotta da Ca-

Tabella. Lo score PRAM e i criteri clinici per la valutazione della severità e della risposta al trattamento del quadro di broncospasmo

\begin{tabular}{|l|l|l|l|l|}
\hline Segni & \multicolumn{2}{l|}{ Punteggio } & $\mathbf{2}$ & $\mathbf{3}$ \\
\hline & $\mathbf{0}$ & $\mathbf{1}$ & Presente & - \\
\hline Retrazione soprasternale & Assente & - & Presente & - \\
\hline Retrazione della muscolatura del collo (scaleni) & Assente & - & Inspiratorio ed espiratorio & $\begin{array}{l}\text { Udibile senza fonendoscopio } \\
\text { o silenzio respiratorio per } \\
\text { ridotto ingresso aereo }\end{array}$ \\
\hline Broncospasmo & Assente & Solo espiratorio & $\begin{array}{l}\text { Udibile senza fonendoscopio } \\
\text { o silenzio respiratorio per } \\
\text { ridotto ingresso aereo }\end{array}$ \\
\hline Ingresso aereo & Normale & Ridotto alle basi & Riduzione diffusa & \\
\hline Saturazione in aria ambiente & & & & $<92 \%$ \\
\hline Il punteggio PRAM si ottiene dalla somma del punteggio per ciascun segno e varia tra 0 (assenza di malattia) e 12 (malattia grave).
\end{tabular}


stro Rodriguez et al. nel 2016 ha invece dimostrato come l'utilizzo dello steroide per via orale in pronto soccorso ed in regime di ricovero possa essere di beneficio nei bambini in età prescolare con frequenti episodi di asma o riacutizzazioni di broncospasmo che richiedono trattamenti urgenti ed ospedalizzazione [5]. Un editoriale che accompagna questa metanalisi suggerisce che l'uso deve essere limitato perché non esistono evidenze che questi farmaci in questa fascia d'età possano dare un beneficio, e il loro uso può essere associato a outcome imprevedibili e eventi avversi [6]. La recente guida GINA indica che l'uso di steroidi per via orale (equivalente a prednisone $1-2 \mathrm{mg} / \mathrm{kg} /$ giorno con un massimo di $20 \mathrm{mg}$ /giorno in bambini $<2$ aa e $30 \mathrm{mg} /$ giorno per bambini tra i 2-5 anni) in età prescolare è raccomandato solo in caso di esacerbazione grave (evidenza forte). Un ciclo di 3-5 giorni è sufficiente e può essere sospeso senza dosaggio a scalare (evidenza debole); la gravità è definita in presenza di almeno 1 di questi segni: paziente agitato, $\mathrm{saO} 2<92 \%$, possibilità di esprimere poche parole, $\mathrm{FC}>180 \mathrm{battiti} / \mathrm{min}(0-3 \mathrm{anni})$ o $\mathrm{FC}>150$ batti$\mathrm{ti} / \mathrm{min}$ (4-5 anni), frequenza respiratoria $>40 \mathrm{atti} / \mathrm{min}$, possibile presenza di cianosi, torace può essere silenzioso [7].

\section{Che cosa aggiunge questo studio}

Questo studio, pur evidenziando uno scarso ruolo della terapia steroidea somministrata per 3 giorni consecutivi sull'outcome primario, conferma l'efficacia dello steroide sul miglioramento del distress respiratorio a 4 e a 24 ore dalla somministrazione sulla riduzione della necessità di ricovero ospedaliero e sulla necessità di terapia steroidea o intravenosa supplementare. Viene suggerito che il trattamento steroideo, se iniziato, dovrebbe essere di breve durata.

\section{Commento}

\section{Validità interna}

Disegno dello studio: la qualità metodologica dello studio è discreta. Tra i punti di forza: la randomizzazione generata in modo adeguato, l'ampio campione con anche variabilità etnica (3 ospedali della Nuova Zelanda), il buon bilanciamento tra i due gruppi al tempo 0 , l'età di inclusione (con esclusione dei bambini $<2$ anni in modo da escludere la bronchiolite e $>5$ anni in modo da escludere l'asma), l'utilizzo del PRAM score per la definizione clinica dei casi e della risposta al trattamento, scala già validata in precedenti studi e dotata di eccellente concordanza tra differenti operatori, ben correlata agli esiti clinici e validata anche in bambini - dai 12 mesi di età). I limiti dello studio, però, sono diversi e rilevanti: 1) il disegno di non inferiorità non sembra giustificato dal quesito di ricerca, ma piuttosto dalla possibilità di reclutare un numero inferiore di pazienti; da segnalare che a studio in corso è stata aumentata la numerosità del reclutamento per raggiungere la disponibilità di dati per l'outcome principale; 2) in uno studio di non inferiorità è necessario, oltre ad una analisi intention to treat, anche un'analisi per protocollo, che includa solo i pazienti che hanno completato lo studio secondo randomizzazione, cosa che in questo lavoro non è stata fatta. Questo indebolisce le conclusioni a cui arrivano gli autori; 2) sono numerosi i persi al follow-up (18\% del totale, ugualmente distribuiti nei due bracci) per quanto riguarda la valutazione di outcome primari e secondari per la difficoltà di contattare i pazienti dimessi. Inoltre numerosi sono i pazienti (564) che hanno rifiutato l'arruolamento e questo potrebbe costituire un bias di selezione; 3) lo steroide per via sistemica viene somministrato indipendentemente dal PRAM score, quando invece ci si aspetterebbe che venisse somministrato solo per i casi moderati gravi. 4) se i medici ritenevano imperativo per la situazione clinica, dopo la terapia broncodilatatoria di attacco, ai pazienti veniva somministrata altra terapia steroidea ed il paziente proseguiva comunque nello studio. Questo però potrebbe inficiare la randomizzazione (i pazienti che ricevevano la terapia steroidea erano coloro che presentavano un quadro più grave).

Esiti: ben definiti e valutati con parametri clinici oggettivi. Non è chiara la rilevanza clinica dell'esito principale: il cambiamento dello score PRAM a 24 ore non è risultato differente tra i 2 gruppi, mentre il valore assoluto dello score sia a 4 che a 24 ore (esiti secondari) è risultato significativamente inferiore nel gruppo prednisolone. Il margine di equivalenza per l'outcome primario è stato scelto dagli autori sulla base di uno studio pilota in 137 casi e probabilmente ha attenuato la differenza tra i trattamenti. Conflitto di interesse: nessuno.

\section{Trasferibilità}

Popolazione studiata: la popolazione studiata è sovrapponibile a quella che accede ai nostri pronti soccorsi e ambulatori di pediatria di base.

Tipo di intervento: l'intervento descritto è realizzabile in Italia.

1. Murphy J., King C., Kapitein B. et al. Oral steroids for preschool children with acute wheeze: a systematic review and meta-analysis. European Respiratory Journal 2019; 54: OA4941; DOI: 10.1183/13993003.

2. Foster S.J., Cooper M. N., Oosterhof S. et al. Oral prednisolone in preschool children with virus-associated wheeze: a prospective, randomised, double-blind, placebo-controlled trial. Lancet Respir Med 2018;6:97-106. DOI: 10.1016/S2213-2600(18)30008-0

3. Panickar J, Lakhanpaul M, Lambert PC et al. Oral prednisolone for preschool children with acute virus-induced wheezing. N Engl J Med. 2009;360(4):329-38 doi: 10.1056/NEJMoa0804897.

4. Beigelman A., Bacharier L.B. Oral corticosteroids in preschool children with severe episodes of virus-associated wheeze: To treat or not to treat? J Allergy Clin Immunol 2018;142(2):405-406. DOI: 10.1016/j. jaci.2018.06.025

5. Castro-Rodriguez JA, Beckhaus AA, Forno E. Efficacy of oral corticosteroids in the treatment of acute wheezing episodes in asthmatic preschoolers: Systematic review with meta-analysis. Pediatr Pulmonol. 2016;51:868-76 DOI: 10.1002/ppul.23429

6. Deshpande D.R., Martinez F. D. The dilemma of systemic steroids in preschool children with recurrent wheezing exacerbations- Pediatr Pulmonol 2016;51(8):775-7. DOI: 10.1002/ppul.23465

7. https://ginasthma.org/gina-reports/. pag 165-168 GINA full report

\section{Scheda redatta dal gruppo di lettura di Milano:}

Riccardo Cazzaniga, Gian Piero Del Bono, Lucia Di Maio, Laura Martelli, Maria Luisa Melzi, Aurelio Nova, Ambrogina Pirola, Giulia Ramponi, Ferdinando Ragazzon, Patrizia Rogari, Alessandra Sala, Federica Zanetto. 